\title{
Table S2A
}

The modifications revealed by the Modiro search engine from HCT data

\begin{tabular}{|c|c|c|}
\hline Spot & CID & ETD \\
\hline 1 & $\begin{array}{l}\text { Substitution: L4H, P277S } \\
\text { 2-amino-3-oxo-butanoic_acid: S140 } \\
\text { Acetylation: S430 } \\
\text { Carbamylation: E1 } \\
\text { Deamidation: N321 } \\
\text { Dihydroxy: R38,T372, T373 } \\
\text { Dimethylation: P33,P34 } \\
\text { Methylation: S7,H291 } \\
\text { Oxidation: M34,M83 }\end{array}$ & $\begin{array}{l}\text { Substitution: E1K, M34V } \\
\text { Carbamylation: E1 } \\
\text { Methylation: H6 }\end{array}$ \\
\hline 2 & $\begin{array}{l}\text { Substitution: N52D, P238T, V48D, C266G, } \\
\quad \text { E264K, D271H, I51N, L320P, N292D, } \\
\quad \text { N303D, N440D } \\
\text { Acetylation: S260 } \\
\text { Amino: Y54, Y155 } \\
\text { Biotinylation: K252 } \\
\text { Deamidation: R19, R38, R87, Q115, R298, } \\
\quad \text { R307, Q317, N321, N390, N395 } \\
\text { Dihydroxy: P233, C235, T266, Y284 } \\
\text { Dimethylation: P129, P233 } \\
\text { Ethylation: K415 } \\
\text { Hydroxylation: P14, N52, N292 } \\
\text { Methylation: S17, S21, T53, K153, E158, S260, } \\
\quad \text { T266,E278, H291, D407, C431, S432, } \\
\text { Oxidation: M4, M34, M83 } \\
\text { Sodiated: E6 }\end{array}$ & $\begin{array}{l}\text { Substitution: D271H, N292D, Q317E, D405H, } \\
\qquad \text { D407H, N440D } \\
\text { Deamidation: Q115, Q317, N321 } \\
\text { Dimethylation: P233 } \\
\text { L-allysine: K280 } \\
\text { Methylation: S21, H274, E278, D407, S409 } \\
\text { Oxidation: M34, M83 } \\
\text { Sodiated: E6 }\end{array}$ \\
\hline 3 & $\begin{array}{l}\text { Substitution: V48D, N52D, K139E, P236T, } \\
\qquad \text { N440D } \\
\text { Acetylation: S432 } \\
\text { Amino: Y284 } \\
\text { Deamidation: R19, Q115, R307, N321, N390 } \\
\text { Dihydroxy: Y284 } \\
\text { Dimethylation: P233, P234 } \\
\text { Ethylation: K415 } \\
\text { Hydroxylation: D318 } \\
\text { L-allysine: K252 } \\
\text { Methylation: S21, E158, E264, E275, E278, } \\
\quad \text { H316, C431, S432, H435 }\end{array}$ & $\begin{array}{l}\text { Substitution: D407H } \\
\text { Amino: Y284 } \\
\text { Deamidation: Q115, N321 } \\
\text { Dihydroxy: Y284 } \\
\text { Hexosamine: T91 } \\
\text { Hydroxylation: D73, P263 } \\
\text { Methylation: S21, E275, K280, H316 } \\
\text { Oxidation: M34 }\end{array}$ \\
\hline
\end{tabular}




\begin{tabular}{|c|c|c|}
\hline & $\begin{array}{l}\text { Oxidation: M83, M335, M434 } \\
\text { Trimethyl-OH: K139 }\end{array}$ & \\
\hline 4 & $\begin{array}{l}\text { Substitution: F429I } \\
\text { Deamidation : N321 } \\
\text { Dihydroxy: Y284 } \\
\text { Hexose : C431 } \\
\text { L-allysine: K139 } \\
\text { Methylation: E6 } \\
\text { Oxidation: M34, M83, M434 }\end{array}$ & $\begin{array}{l}\text { Dihydroxy: Y284 } \\
\text { Hydroxylation: P263 } \\
\text { Oxidation :M34 }\end{array}$ \\
\hline 5 & $\begin{array}{l}\text { Substitution: D120H,D151H, D1H, D1N, } \\
\text { D28H, I117N, M4R, T5R, N137D, } \\
\text { N31D, N34D, Q155E, S30A, V196D, } \\
\text { K126E } \\
\text { Acetylation: D1 } \\
\text { Carbamylation: D1, K42, K45, K149 } \\
\text { Deamidation: R18, R142, Q199 } \\
\text { Dihydroxy: T5, T20, T22, Y32, T197 } \\
\text { Dimethylation: P204 } \\
\text { GlyGLy: K190 } \\
\text { Hydroxylation: D1 } \\
\text { Hexosamine: T51, N210 } \\
\text { Hexose: K183 } \\
\text { Methylation: H55, S121, D122, Q124, S127, } \\
\quad \text { Q160, E161, E165, E195 } \\
\text { N-pyruvic acid 2-iminyl: K190 } \\
\text { Phosphorylation: S127 } \\
\text { Pyruvat: C23 }\end{array}$ & $\begin{array}{l}\text { Substitution: D1N } \\
\text { Carbamylation: D1, K45 } \\
\text { Deamidation: R108, R142, Q199 } \\
\text { Dihydroxy: T197 } \\
\text { Methylation: H55, E165 } \\
\text { Oxidation: M4 }\end{array}$ \\
\hline 6 & $\begin{array}{l}\text { Substitution: D122H, D151H, D170N, D1H, } \\
\quad \text { D1N, I117N, I29N, I48M, L154F, D167H, } \\
\quad \text { K126E } \\
\text { Acetylation: K190 } \\
\text { Amino: Y192 } \\
\text { Carboxyethyl: K149 } \\
\text { Dihydroxylation: T197 } \\
\text { Deamidation: R18, Q27, N34,R 108, N137, } \\
\quad \text { R142, Q199 } \\
\text { Hexosamine: T51 } \\
\text { Hydroxylation: D1 } \\
\text { L-allysine: K39 } \\
\text { Methylation: S9, S10, Q27, N31, N34, S52, } \\
\quad \text { S53, H55, S121, D122, E123, S127, } \\
\quad \text { S131, S156, N158, E165, E195 } \\
\text { Nitro: Y186, Y192 } \\
\text { Oxidation: M4 }\end{array}$ & $\begin{array}{l}\text { Substitution: A193E, D1N } \\
\text { Deamidation: R108, R142, Q199 } \\
\text { Methylation: S52, S53, S56, T164 } \\
\text { Oxidation: M4 }\end{array}$ \\
\hline
\end{tabular}




\begin{tabular}{|l|l|l|}
\hline 7 & Trimethyl-OH: K190 & \\
\hline & $\begin{array}{l}\text { Substitution: D1H, D1N, D28H, D122H, I48N, } \\
\text { D151H, D170H, D170N }\end{array}$ & $\begin{array}{l}\text { Substitution: V191G } \\
\text { Deamidation: R285 } \\
\text { Amino: Y192 } \\
\text { Deamidation: Q3, R18, Q27, N31, N34, N137, } \\
\text { Q199 } \\
\text { Dihydroxylation: Y36, P44, K45, Y49, K190, } \\
\text { T197 } \\
\text { Diphthamide: H189 } \\
\begin{array}{l}\text { Hydroxylation: K39 } \\
\text { Methylation: Q3, Q6, S7, Q27, T51, E123, } \\
\text { S127, E165, E195, Q199 }\end{array} \\
\\
\text { Oxidation: M4 }\end{array}$ \\
\hline
\end{tabular}


Table S2B

The modifications revealed by the Modiro search engine from Orbitrap data

\begin{tabular}{|c|c|}
\hline Spot & Modification \\
\hline 2 & $\begin{array}{l}\text { Substitution: N440D, L320Q, N367D, G287D, N395D, N292D, G322D, F29I, } \\
\quad \text { G144D, C230G, S85N, A333E, G149D, A79S, A97E, N331D, G322D, } \\
\text { Y94D, F70Y, L320Q, N303D, N292D, Q317E, K346Q, F70Y, M258T, } \\
\quad \text { G149D, A97E, N331D, F70Y, T372N, S85N, F70Y, N303D } \\
\text { Acetylation: S140 } \\
\text { Carbamylation: K139, K224, K366, K420 } \\
\text { Deamidation: R87, N321, N390 } \\
\text { Dihydroxy: S7, Y32, R38, Y80, Y94, K153, P188, Y284, K323, S389, S430 } \\
\text { GlyGLy: K332, , K420 } \\
\text { Hexose: K139, R289 } \\
\text { Hydroxylation: N282, P234, N390 } \\
\text { Kynurenin: W36, W283, W319 } \\
\text { L-allysine: K366 } \\
\text { Methylation: E6, E89, K98, T266, E264, E275, E289, E299, T372 } \\
\text { Nitro: Y27, Y80, Y355, Y413 } \\
\text { Oxidation: M22, M34, M83, M258, M364, M434 } \\
\text { Sulphone: M34 }\end{array}$ \\
\hline 4 & $\begin{array}{l}\text { Substitution: N440D, L320Q, N367D, G287D, N395D, N292D, G322D, F29I, } \\
\quad \text { G144D, C230G, S85N, A333E, G149D, A79S, A97E, N331D, G322D, } \\
\text { Y94D, F70Y, L320Q, N303D, N292D, Q317E, K346Q, F70Y, M258T, } \\
\quad \text { G149D, A97E, N331D, F70Y, T372N, S85N, F70Y, N303D } \\
\text { Acetylation: K328 } \\
\text { Carbamylation: K332, K340 } \\
\text { Deamidation: R87, N321 } \\
\text { Dihydroxy: R87, Y284 } \\
\text { GlyGLy: K98, K42 } \\
\text { Hexose: K98 } \\
\text { Hydroxylation: D73, N282 } \\
\text { Kynurenin: W283 } \\
\text { Methylation: E6, E89, E289, T372 } \\
\text { Oxidation: M34, M83, M258, M364 } \\
\text { Sulphone: M83 }\end{array}$ \\
\hline 6 & $\begin{array}{l}\text { Substitution: K207R, T197S, F50Y, N158D, L154S, L154Q, N137D, T197S, } \\
\text { R19K, N34D, Q199R, T197S, F50Y, N158K, N137D, R18K } \\
\text { Amino: Y192 }\end{array}$ \\
\hline
\end{tabular}




\begin{tabular}{|l|l|}
\hline & Acetylation: S7, K149, K207 \\
Carbamylation: K207 \\
Deamidation: R142, Q199 \\
Dihydroxy: Y36,S56,K149,S156,S208, \\
GlyGLy: K190, K207 \\
Hexose: W148, K183 \\
Hydroxylation: D122, N137, K183 \\
L-allysine: K207 \\
Methylation: S121, D122, E123, E165, E195 \\
Nitro: Y36, Y49, Y140,Y173, Y192 \\
Oxidation M4 \\
Sulphone: M4 \\
Substitution: K207R, T197S, F50Y, N158D, L154S, L154Q, N137D, T197S, \\
R19K, N34D, Q199R, T197S, F50Y, N158K, N137D, R18K \\
Acetylation: K149,K190,K207 \\
Carbamylation: K190, K207 \\
Deamidation: R142, Q199 \\
Dihydroxy: Y36, K149, T197 \\
GlyGLy: K190 \\
Hexose: K183 \\
Hydroxylation: K39, N137, D170, K190 \\
L-allysine: K207 \\
Methylation: E123, E165, E195 \\
Nitro: Y49, Y173, Y192 \\
Oxidation: M4 \\
Sulphone: M4
\end{tabular}

